\title{
Glaucoma and mortality in the Beijing Eye Study
}

\begin{abstract}
Purpose To assess the relationship between glaucoma and mortality in a population-based setting.

Methods At baseline in 2001, the Beijing Eye
\end{abstract} Study examined 4356 subjects for glaucoma with a detected glaucoma frequency of 135/ 4356 or $3.1 \%$. Mean age was $55.9 \pm 10.4$ years (40-101 years). In 2006, all study participants were reinvited for a follow-up examination. Results Out of the 4356 subjects, 3208 (73.6\%) subjects returned for follow-up examination, while 124 (2.8\%) subjects were dead, and 1024 (23.5\%) subjects did not agree to be reexamined or had moved away. Mortality rate was significantly $(P<0.001$; odds ratio (OR):

${ }^{1}$ Beijing Institute of Ophthalmology, Beijing Tongren Hospital, Capital University of Medical Science, Beijing, China

${ }^{2}$ Department of Ophthalmology, Faculty of Clinical Medicine Mannheim, University of Heidelberg, Mannheim, Germany

Correspondence: $\mathrm{L} X \mathrm{X}$ or JB Jonas,

Beijing Institute of

Ophthalmology,

17 Hougou Street,

Chong Wen Men,

Beijing 100005, China

Tel: +49621383 2652;

Fax: +49621383 3803 .

E-mails: xuliang5918@

yahoo.com.cn

or Jost.Jonas@augen.ma.

uni-heidelberg.de

Received: 11 April 2007 Accepted in revised form: 24 November 2007

Published online: 11 January 2008

Proprietary interest: None 4.72; 95\% confidence interval (CI): $2.67,8.33$ ) higher in the 135 glaucoma subjects $(15 / 135$ or $11.1 \pm 2.7 \%$; 95\% CI: $5.8,16.4)$ than in the 4221 participants without glaucoma (109/4221 or $2.6 \pm 0.2 \%$; 95\% CI: $2.2,3.0$ ). In binary logistic regression analysis, mortality was significantly associated with age $(P<0.001)$, gender ( $P<0.001$; OR: $0.44 ; 95 \%$ CI: $0.29,0.66)$, level of education $(P<0.001$; OR: $0.64 ; 95 \%$ CI: 0.55 , $0.74)$, and the presence of glaucoma $(P=0.007$; OR: 2.30; 95\% CI: $1.26,4.20)$. If the whole glaucoma group was differentiated into an open-angle glaucoma group and an angleclosure group, mortality was still significantly associated with age $(P<0.001)$, gender $(P<0.001)$, level of education $(P<0.001)$, and with the presence of angle-closure glaucoma $(P=0.006$; OR: 3.09 ; 95\% CI: $1.49,10.2)$, while the association with the presence of openangle glaucoma was marginally significant $(P=0.13$; OR: $1.83 ; 95 \%$ CI: $0.84,4.01)$.

Conclusions The data suggest that glaucoma, particularly angle-closure glaucoma, may be associated with an increased rate of mortality in adult Chinese in Greater Beijing. Eye (2008) 22, 434-438; doi:10.1038/sj.eye.6703072; published online 11 January 2008

Keywords: glaucoma; mortality; Beijing eye study
$\mathrm{LXu}^{1}, \mathrm{YX}$ Wang $^{1}$ and JB Jonas ${ }^{1,2}$

\section{Introduction}

For any age-related disease such as the glaucoma, the question arises whether they are associated with, or independent of, an increased mortality of the patient. ${ }^{1-11}$ It may particularly hold true, if systemic vascular factors for the glaucoma are discussed to play a pathogenic role. ${ }^{12,13}$ It was, therefore, the purpose of the present population-based investigation to assess whether glaucoma is related to an increased mortality of the affected subjects.

\section{Methods}

The Beijing Eye Study is a population-based study in northern China. It was carried out in four communities in the urban district of Haidian in the north of Central Beijing and in three communities in the village area of Yufa of the Daxing District in the south of Beijing. The study has been described in detail recently. ${ }^{14-16}$ The Medical Ethics Committee of the Beijing Tongren Hospital had approved the study protocol and all participants had given informed consent, according to the Declaration of Helsinki. At the time of the first survey in 2001, the seven communities had a total population of 5324 individuals aged 40 years or above. In total, 4439 individuals (2505 women) participated in the eye examination, corresponding to an overall response rate of $83.4 \%$. The study was divided into a rural part (3946 eyes, 1973 subjects; 1143 women) and an urban part (4932 eyes, 2466 subjects; 1362 women). Mean age was $56.20 \pm 10.59$ years (median, 56 years; range, 40-101 years).

In 2006, the study was repeated by reinviting all participants from the 2001 survey to be re-examined with 3251 subjects participating (response rate: $73.3 \%$ ). All examinations were carried out in the communities, either in schoolhouses or in community houses. Uncorrected visual acuity was measured (Snellen charts) at a distance of $5 \mathrm{~m}$. Automatic 
refractometry (Auto Refractometer AR-610; Nidek Co., Ltd, Tokyo, Japan) was performed, when uncorrected visual acuity was lower than 1.0. Visual field examinations were performed by the frequency-doubling perimetry using the screening program C-20-1 (ZeissHumphrey, Dublin, CA, USA). ${ }^{17,18}$ Intraocular pressure was measured using a noncontact pneumotonometer (CT-60 computerized tonometer; Topcon Ltd, Japan) by an experienced technician. Three measurements were taken, and the mean of the three measurements was taken for further statistical analysis. If the measurements were higher than $25 \mathrm{mmHg}$, tonometry was repeated. A slit-lamp examination was carried out by an ophthalmologist. The pupil was dilated using tropicamide once or twice, until the pupil diameter was at least $6 \mathrm{~mm}$. Digital photographs of the cornea and retro-illuminated photographs of the lens were taken using the Neitz CT-R camera (Neitz Instruments Co., Tokyo, Japan). Monoscopic photographs (on film) of the macula and optic disc were taken using a fundus camera (Type CR6-45NM; Canon Inc., USA). Central corneal thickness was measured by slit-lamp-mounted optical coherence tomography (Heidelberg Engineering Co., Heidelberg, Germany). Past history of eye diseases, eye trauma, diabetes mellitus, hypertension, and any ophthalmologic care the participant received were recorded. Further information was received by a questionnaire, which included questions on family income. In addition to the eye examinations, body weight and height were measured.

The optic disc slides taken in 2001 were projected, and we examined the qualitative parameters, 'shape of the neuroretinal rim' with special respect to the InferiorSuperior-Nasal-Temporal (ISNT) rule, including the presence of neuroretinal rim notches, occurrence of optic disc hemorrhages, presence of localized defects in the retinal nerve fibre layer, decreased diffuse visibility of the retinal nerve fibre layer, and the occurrence of marked diffuse thinning or focal thinning of the retinal arteries in the peripapillary region. ${ }^{19}$ The optic disc photographs of all eyes with suspicious optic nerve heads, of all highly myopic eyes, of all eyes with an intraocular pressure higher than $21 \mathrm{mmHg}$, of all eyes with a visual field loss and of all eyes with a visual acuity of less than 0.50 were separately re-reviewed twice by a panel (including LX and JBJ). The panel usually consisted of the whole junior research staff of the Beijing Institute of Ophthalmology, including up to 10 residents plus staff members including LX and JBJ. The panel reviewed more than 3000 slides mainly of the optic disc and the macula.

For the definition of glaucoma ('optic disc glaucoma'), the only criterion for glaucoma was a glaucomatous appearance of the optic disc. ${ }^{16}$ Absolute criteria for a glaucomatous appearance of the optic nerve head, each of which were sufficient for the diagnosis of glaucoma, were a notch in the neuroretinal rim in the temporal inferior region and/or the temporal superior region, so that the ISNT rule was not fulfilled (in eyes with an optic cup sufficiently large to allow an assessment of the neuroretinal shape); a localized retinal nerve layer defect which could not be explained by any cause other than glaucoma; and an abnormally large cup in relation to the size of the optic disc. ${ }^{19}$ The relative criteria for the diagnosis of a glaucomatous appearance of the optic nerve head were if the neuroretinal rim was markedly thinner in inferior disc region compared with the superior disc region, even if the smallest part of the neuroretinal rim was located in the temporal horizontal disc region; a diffuse decrease in the visibility of the retinal nerve fibre layer (particularly in eyes with small optic discs), if the background pigmentation of the eye allowed an assessment of the retinal nerve fibre layer and if there were no other reasons than glaucoma for retinal nerve fibre layer loss; a marked diffuse thinning and/or focal thinning of the retinal arteries if there were no other reasons than glaucoma for retinal vessel thinning; and an optic disc haemorrhage, if there were no other reasons for a disc bleeding such as retinal vessel occlusions. If none of the absolute glaucoma criteria were positive, at least two relative criteria had to be positive, including a suspicious neuroretinal rim shape in eyes with an optic cup large enough for the assessment of the rim shape; or at least two relative criteria had to be positive, including the occurrence of an optic cup in a small optic disc which usually would not show cupping. The height of intraocular pressure and presence of visual field defects were no criteria for the diagnosis of optic disc glaucoma.

Open-angle glaucoma was defined by an open anterior chamber angle and a normal depth of the anterior chamber as assessed by slit-lamp biomicroscopy and gonioscopy. Gonioscopy was performed using a Goldmann gonioscope by the same glaucoma expert. In angle-closure glaucoma, the anterior chamber angle was occluded, or there was appositional contact between the peripheral iris and the cornea, for at least 15, that is, Schwalbe's line could not be visualized. In addition, other features for angle-closure glaucoma were peripheral anterior synechiae, iris whirling (distortion of the radially orientated iris fibres), and glaukomflecken in the anterior subcapsular lens region, in combination with a narrow anterior chamber angle. This definition is partially different from the definition by Foster et al, ${ }^{20}$ who defined an angle as occludable if $\geqslant 270^{\circ}$ of the posterior trabecular meshwork (the part which is often pigmented) cannot be seen upon gonioscopy. At the first glance, Foster's definition and the one used in the present study appear to be different. If, however, Schwalbe's line is not at all visible at one part of the 
anterior chamber angle, the whole anterior chamber is usually markedly narrowed so that the pigmented part of the trabecular meshwork is usually not visible for $\frac{3}{4}$ of the circumference. It may, therefore, suggest that both definitions of an occludable angle may not be markedly different.

Statistical analysis was performed using a commercially available statistical software package (SPSS for Windows, version 15.0, SPSS, Chicago, IL, USA). The data on frequencies are given as mean $\pm S E$, all other data on the means are presented as mean \pm SD. Logistic regression was used to investigate the associations of the binary dependent variable mortality with the continuous or categorical independent variables, such as age, gender, and the presence of glaucoma. Ninety-five percent confidence intervals (CI) and odds ratios (ORs) were presented. All $P$-values were two-sided and were considered statistically significant when the values were less than 0.05 .

\section{Results}

From the 4439 individuals participating in the study in 2001, readable optic disc photographs were available for $4356(98.1 \%)$ subjects with $1892(43.4 \%)$ subjects from the rural region and $2464(56.6 \%)$ subjects from the urban region entered into the statistical analysis. The mean age was $55.9 \pm 10.4$ years (median, 56 years; range, 40-101 years), and the mean refractive error was $-0.38 \pm 2.29 \mathrm{D}$ (median, 0D; range, $-20.03 \mathrm{D}$ to $+13.50 \mathrm{D}$ ). A glaucomatous appearance of the optic disc (optic disc glaucoma) was detected in $220(2.5 \%)$ eyes of $135(3.1 \%)$ subjects. Out of the 135 glaucoma subjects with glaucomatous optic nerve damage, $96(71 \%)$ were classified as open-angle glaucoma, and 39 (29\%) as angle-closure glaucoma. In 2006, all study participants were reinvited for a follow-up examination.

Out of the 4356 subjects, 3208 (73.6\%) subjects returned for the follow-up examination, while 124 (2.8\%) subjects were dead and 1024 (23.5\%) subjects did not agree to be re-examined or had moved away. Mortality rate was significantly $(P<0.001$; OR: $4.72 ; 95 \% \mathrm{CI}: 2.67,8.33)$ higher in the 135 glaucoma subjects $(15 / 135$ or $11.1 \pm 2.7 \%$; $95 \%$ CI: $5.8,16.4)$ than in the 4221 participants without glaucoma (109/4221 or $2.6 \pm 0.2 \%$; 95\% CI: $2.2,3.0)$. In univariate analysis, mortality rates were significantly correlated with age $(P<0.001 ;$ OR: 1.08; 95\% CI: $1.06,1.10)$, low family income $(P<0.001)$, level of education $(P<0.001$; OR: $0.61 ; 95 \%$ CI: 0.54, 0.69), gender $(P=0.004$; OR: 0.58 ; 95\% CI: $0.41,0.84)$, and glaucoma $(P<0.001$; OR: 4.72 ; 95\% CI: $2.67,8.33)$.

A binary logistic regression analysis, with the occurrence of death as the dependent variable, and age, gender, family income, and the presence of glaucoma as independent variables, revealed that mortality was still significantly associated with age $(P<0.001 ;$ OR: $1.08 ; 95 \%$ CI: $1.07,1.11)$, gender $(P<0.001$; OR: $0.47 ; 95 \%$ CI: 0.32 , $0.69)$, low family income $(P<0.001)$, and the presence of glaucoma $(P=0.005$; OR: $2.43 ; 95 \% \mathrm{CI}: 1.32,4.48)$. If the level of education instead of family income was taken as independent parameter in the regression analysis, a similar result was obtained, with mortality being significantly associated with age $(P<0.001 ;$ OR: $1.06 ; 95 \%$ CI: 1.04, 1.08), gender $(P<0.001$; OR: $0.44 ; 95 \%$ CI: 0.29 , $0.66)$, level of education ( $P<0.001$; OR: $0.64 ; 95 \%$ CI: 0.55 , $0.74)$, and the presence of glaucoma ( $P=0.007$; OR: 2.30; $95 \%$ CI: $1.26,4.20)$. If only subjects with an age of less than 65 years were included in the statistical analysis, the results were mostly unchanged, with a significant association between the presence of glaucoma and increased mortality $(P=0.007$; OR: $4.15 ; 95 \% \mathrm{CI}$ : 1.49, 11.6).

To further elucidate the dependence of mortality on age, a new parameter was formed as a product of age and presence of glaucoma. Including this parameter into the multivariate analysis revealed that mortality was still significantly associated with age $(P<0.001 ;$ OR: $1.07 ; 95 \%$ CI: $1.03,1.11)$, gender $(P<0.001$; OR: 0.36 ; 95\% CI: 0.20 , $0.62)$, level of education ( $P<0.001$; OR: $0.63 ; 95 \%$ CI: 0.50 , $0.79)$, and the presence of glaucoma $(P=0.02$; OR: 7.07; 95\% CI: $1.33,37.6)$.

If the whole glaucoma group was differentiated into an open-angle glaucoma group and an angle-closure group and using the same binary logistic regression, mortality was still significantly associated with age $(P<0.001$; OR: 1.06; 95\% CI: $1.04,1.08)$, gender $(P<0.001$; OR: 0.43 ; 95\% CI: $0.28,0.65)$, level of education $(P<0.001$; OR: $0.62 ; 95 \%$ CI: $0.53,0.72)$, and marginally significant with the presence of open-angle glaucoma $(P=0.13$; OR: $1.83 ; 95 \%$ CI: $0.84,4.01)$. In a similar manner, including only eyes with angle-closure glaucoma or non-glaucomatous eyes revealed that mortality was significantly associated with age $(P<0.001$; OR: 1.06 ; 95\% CI: 1.04, 1.08), gender $(P=0.001$; OR: $0.49 ; 95 \%$ CI: $0.32,0.76)$, level of education $(P<0.001$; OR: $0.70 ; 95 \% \mathrm{CI}: 0.59,0.82)$, and with the presence of angle-closure glaucoma ( $P=0.006$; OR: 3.09; 95\% CI: $1.49,10.2)$.

\section{Discussion}

The results suggest that glaucoma was associated with an increased mortality in adult Chinese during a follow-up of 5 years in Greater Beijing. It is in agreement with a previous report from the Blue Mountains Eye Study in which an increased cardiovascular mortality in persons with previously diagnosed glaucoma was found. ${ }^{6}$ It is also in agreement with a previous study 
by Lee et $a l,{ }^{21}$ who found in a National Health Interview Survey from 1986 to 1994 that among adults residing in the United States, reported glaucoma was associated with an increased risk of all-cause and cardiovascular disease mortality. The results of the present study are parallel to reports that retinal vein occlusions are associated with an increased mortality. ${ }^{10,11}$ The present study is partially in disagreement with a Swedish study by Godrum $e t a l^{3}$ in which open-angle glaucoma patients, newly detected in a population-based screening of more than 32000 elderly citizens or seen in routine clinical practice, did not differ significantly in the 5 -year mortality from control groups of the same age and gender.

There are limitations in the present study, one of which may be the definition of glaucoma. Since marked glaucomatous optic nerve damage can be present if the visual field examination is normal, we did not use a perimetric defect as additional criterion for the definition of optic disc glaucoma. In a similar manner, intraocular pressure was not taken into account since subjects with so-called normal-pressure glaucoma would not have been considered to have glaucoma. The assessment of the optic nerve head appearance was subjective. To overcome this weakness of the study, all photographs of all eyes with suspicious optic nerve heads, of all highly myopic eyes, of all eyes with an intraocular pressure higher than $21 \mathrm{mmHg}$ and of all eyes with any visual field loss or a visual acuity of less than 0.50 were separately re-reviewed by a panel, including two glaucoma experts. In addition, the photographs of all eyes with an intraocular pressure higher than $21 \mathrm{mmHg}$ and the photographs of all eyes with the diagnosis of glaucoma were re-checked twice by a panel to confirm the diagnosis. Inspite of these multiple steps, however, there may be underdiagnosed glaucomatous eyes in the group not being repeatedly re-checked, and there is the possibility of an overdiagnosis of glaucoma in the repeatedly examined group, despite an assessment by a panel. Another limitation of the study is that the glaucoma definition used in the present study is not identical to the definition used in other population-based studies. ${ }^{20}$ This may be a principal flaw in the study design and may make the comparison of the prevalence data difficult. On the other hand, however, the question may arise how sensitive and specific each of the definitions are. It has been shown in hospital-based studies that quantitative optic nerve head parameters, such as neuroretinal rim area, cup/disc ratios corrected for their dependence on the optic disc size, and semiquantitative parameters of the visibility of the retinal nerve fibre layer are inferior in their diagnostic precision to detect glaucoma when compared with qualitative parameters, such as the presence or absence of neuroretinal rim notches, whether the ISNT rule is fulfilled or not, the presence of an optic disc haemorrhage or the presence of a localized retinal nerve fibre layer. ${ }^{19}$ The advantage of these qualitative optic nerve head parameters of a relatively high diagnostic precision is combined with the disadvantage that they have to be assessed by an individual examiner introducing an intra- and an inter-observer variability. Another potential weakness of the study design is the use of pneumotonometry. However, as intraocular pressure was not taken for the diagnosis of glaucoma, this potential weakness of the study may not have influenced the major outcome parameter of the study, that is, the prevalence of glaucoma. A further limiting factor may have been that an elevated intraocular pressure measurement was a criterion for the re-checking of the optic disc slides, so that eyes with an elevated intraocular pressure may have had a higher chance to be detected as glaucomatous. It could have led to an increased ratio of angle-closure glaucoma to open-angle glaucoma. However, as the proportion of angle-closure glaucoma to open-angle glaucoma in the present study was equal to or lower than in other investigations, it may only serve to strengthen that finding. ${ }^{22-28}$ Another limitation of the study is that gonioscopy was performed only for all study participants with glaucoma and all glaucoma suspects. However, as some of the glaucoma suspects were discovered as such first at the occasion of the assessment of the optic disc photographs, which took later than the clinical examination, gonioscopy was not performed for all glaucoma suspects. For these subjects, the assessment of the anterior chamber depth as had been performed by slit-lamp biomicroscopy using van Herick's method was taken to semiquantify the anterior chamber angle anatomy. Another limitation is that the use of van Herick's assessment of the angle depth is highly subjective with a usually low negative predictive rate. In case of doubt, it would, therefore, have resulted in a falsely high rate of angle-closure glaucoma. As, however again, the proportion of angle-closure glaucoma to openangle glaucoma was relatively low in the present study compared with other investigations, it may only serve to strengthen the assessment. Another limitation of the study is that as the causes of death had not been investigated usually due to lack of infrastructure, the death causes could not be included in the statistical analysis. The study is, therefore, unable to provide information on whether the various death causes are associated with other parameters.

In conclusion, the present study suggests that in adult Chinese in Greater Beijing, glaucoma, particularly angleclosure glaucoma, may be associated with an increased rate of mortality. 


\section{Acknowledgements}

This study was supported by the Beijing Key Laboratory Fund and the Beijing Natural Science Foundation.

\section{References}

1 Cugati S, Cumming RG, Smith W, Burlutsky G, Mitchell P, Wong JJ. Visual impairment, age-related macular degeneration, cataract, and long-term mortality: the Blue Mountains Eye Study. Arch Ophthalmol 2007; 125: 917-924.

2 Wang JJ, Mitchel P, Simpson JM, Cumming RG, Smith W. Visual impairment, age-related cataract, and mortality. Arch Ophthalmol 2001; 119: 1186-1190.

3 Godrum K, Heijl A, Bengtsson B. Glaucoma and mortality. Graefes Arch Clin Exp Ophthalmol 2004; 242: 397-401.

4 Clemons TE, Kurinii N, Sperduto RD, AREDS Research Group. Associations of mortality with ocular disorders and an intervention of high-dose antioxidants and zinc in the Age-Related Eye Disease Study: AREDS report no. 13. Arch Ophthalmol 2004; 122: 716-726.

5 Knudtsson MD, Kein BE, Klein R. Age-related eye disease, visual impairment, and survival: the Beaver Dam Eye Study. Arch Ophthalmol 2006; 124: 243-249.

6 Lee AJ, Wang JJ, Kifley A, Mitchell P. Open-angle glaucoma and cardiovascular mortality: the Blue Mountains Eye Study. Ophthalmology 2006; 113: 1069-1076.

7 Wang JJ, Cugati S, Knudtson MD, Rochtchina E, Klein R, Klein BE et al. Retinal arteriolar emboli and long-term mortality: pooled data analysis from two older populations. Stroke 2006; 327: 1833-1836.

8 Tan JS, Mitchell P, Smith W, Wang JJ. Cardiovascular risk factors and the long-term incidence of age-related macular degeneration: the Blue Mountains Eye Study. Ophthalmology 2007; 114: 1143-1150.

9 Wong TY, Tikellis G, Sun C, Klein R, Couper DJ, Sharret AR. Age-related macular degeneration and risk of coronary heart disease: the atherosclerosis risk in Communities Study. Ophthalmology 2007; 114: 86-91.

10 Cugati S, Wang JJ, Knudtson MD, Rochtchina E, Klein R, Klein BE et al. Retinal vein occlusion and vascular mortality pooled data analysis of 2 population-based cohorts. Ophthalmology 2006; 114: 520-524.

$11 \mathrm{Xu}$ L, Liu W, Wang Y, Yang H, Jonas JB. Retinal vein occlusions and mortality. The Beijing Eye Study. Am J Ophthalmol 2007; 114: 972-973 in press.

12 Flammer J, Orgul S, Costa VP, Orzalesi N, Krieglstein GK, Serra LM et al. The impact of ocular blood flow in glaucoma. Prog Retin Eye Res 2002; 21: 359-393.

13 Hayreh SS. Blood flow in the optic nerve head and factors that may influence it. Prog Retin Eye Res 2001; 20: 595-624.
14 Wang $\mathrm{Y}, \mathrm{Xu} \mathrm{L}$, Jonas JB. Prevalence and causes of visual field loss as determined by frequency doubling perimetry in urban and rural adult Chinese. Am J Ophthalmol 2006; 141: 1078-1086.

15 Wang Y, Xu L, Zhang L, Yang H, Ma Y, Jonas JB. Optic disc size in a population-based study in Northern China. The Beijing Eye Study. Br J Ophthalmol 2006; 90: 353-356.

16 Xu L, Wang Y, Wang S, Wang Y, Jonas JB. High myopia and glaucoma susceptibility. The Beijing Eye Study. Ophthalmology 2007; 114: 216-220.

17 Thomas D, Thomas R, Muliyil JP, George R. Role of frequency doubling perimetry in detecting neuroophthalmic visual field defects. Am J Ophthalmol 2001; 131: 734-741.

18 Keltner JL, Johnson CA, Cello KE, Edwards MA, Bandermann SE, Kass MA et al. Classification of visual field abnormalities in the ocular hypertension treatment study. Arch Ophthalmol 2003; 121: 643-650.

19 Jonas JB, Budde WM, Panda-Jonas S. Ophthalmoscopic evaluation of the optic nerve head. Surv Ophthalmol 1999; 43: 293-320.

20 Foster PJ, Buhrmann R, Quigley HA, Johnson GJ. The definition and classification of glaucoma in prevalence surveys. Br J Ophthalmol 2002; 86: 238-242.

21 Lee DJ, Gómez-Marín O, Lam BL, Zheng DD. Glaucoma and survival: the National Health Interview Survey 1986-1994. Ophthalmology 2003; 110: 1476-1483.

$22 \mathrm{Hu}$ CZ. An epidemiologic study of glaucoma in Shunyi County, Beijing. Zhonghua Yan Ke Za Zhi 1989; 25: 115-119.

23 Foster PJ, Baasanhu J, Alsbirk PH, Munkhbayar D, Uranchimeg D, Johnson GJ. Glaucoma in Mongolia-a population-based survey in Hovsgol Province, Northern Mongolia. Arch Ophthalmol 1996; 114: 1235-1241.

24 Jacob A, Thomas R, Koshi SP, Braganza A, Muliyil J. Prevalence of primary glaucoma in an urban south Indian population. Indian J Ophthalmol 1998; 46: 81-86.

25 Foster PJ, Oen FT, Machin D, Munkhbayar D, Uranchimeg D, Johnson GJ. The prevalence of glaucoma in Chinese residents of Singapore. Arch Ophthalmol 2000; 118: 1105-1111.

26 Dandona L, Dandona R, Mandal P, Srinivas M, John RK, McCarty CA et al. Angle Closure of glaucoma in an urban population in southern India. Ophthalmology 2000; 107: 1710-1716.

27 Ramakrishnan R, Nirmalan PK, Krishnadas R, Thulasiraj $\mathrm{RD}$, Tielsch JM, Katz J et al. Glaucoma in a rural population of southern India: the Aravind comprehensive eye survey. Ophthalmology 2003; 110: 1484-1490;erratum in Ophthalmology 2004; 111: 331

28 He M, Foster PJ, Ge J, Huang W, Zheng Y, Friedman DS et al. Prevalence and clinical characteristics of glaucoma in adult Chinese: a population-based study in Liwan District, Guangzhou. Invest Ophthalmol Vis Sci 2006; 47: 2782-2788. 